\title{
Homozygosity for autosomal dominant Marfan syndrome
}

\author{
JUAN CHEMKE*, RACHEL NISANI*, AMI FEIGL†, RIVKA GARTY \\ MICHAEL COOPER , YEHUDIT BARASH†, AND DAN DUKSIN \\ From the *Clinical Genetics Unit, $\dagger$ Heart Institute, and $\ddagger$ Department of Pediatrics B, Kaplan Hospital \\ (affiliated to the Hebrew University and Hadassah School of Medicine); and §Department of \\ Biophysics, Weizmann Institute of Science, Rehovot, Israel.
}

SUMMARY Marfan syndrome is an autosomal dominant condition with varying phenotypic manifestations. Affected persons are usually heterozygotes. A family is presented in which the gene for this syndrome is segregating in a large number of members. Two sibs suffered from unusually severe, identical, and fatal manifestations from birth, their parents having mild cardiovascular and somatic symptoms common in Marfan syndrome. Investigation of collagen biosynthesis in fibroblasts revealed no abnormalities in fibronectin and procollagen I and III synthesis and secretion or in the procollagen to collagen conversion. We suggest that these two sibs are examples of homozygosity for the Marfan syndrome gene, based on the large number of affected members, the absence of additional consanguinity, manifestation of the syndrome in both parents, and the severity of the disease in the two sibs.

Marfan syndrome is one of the more frequent autosomal syndromes. Its frequency has been estimated at 4 to 6 in 100000 births. Approximately $15 \%$ of the cases are spontaneous mutations. ${ }^{1}$ There is great variability of the phenotypic manifestations of the disease which may be explained in part by variable penetrance of the gene or genetic heterogeneity. ${ }^{2}$ As in most autosomal dominant diseases, affected subjects are usually heterozygous for the mutant gene.

We report a family in which the gene for Marfan syndrome is segregating. In this kindred, a large number of persons have different manifestations of the disease. Two sibs are described in detail, with identical uncommonly severe manifestations from birth. These patients raise the possibility of homozygosity for the Marfan syndrome gene. The possibility of an autosomal recessive 'Marfan-like' disease of connective tissue cannot be definitely ruled out at present, since so far no specific abnormal collagen for Marfan syndrome is known.

\section{Family history and case reports}

The family pedigree is presented in fig 1 . The family is of Jewish-Yemenite origin. The parents (III.3 and

Received for publication 19 April 1983. Accepted for publication 7 October 1983.
III.4) of the proband (IV.1) and his sister (IV.2) are first cousins. They are tall compared with other members of the family and considering their ethnic origin. Their only skeletal manifestation of Marfan syndrome is a moderately excavated sternum. They both have elinical and echocardiographic evidence of cardiovascular disease: mitral prolapse and aortic ectasia (III.3) and mitral prolapse only (III.4) (fig 2). Ophthalmological examination was normal. The pedigree also shows that a large number of family members have at least two major manifestations of Marfan syndrome, such as arachnodactyly, chest deformities, scoliosis with loose joints, and aortic insufficiency or a floppy mitral valve or both.

The two sibs (IV.1 and IV.2) had progressively severe manifestations from birth and died in early infancy. Their pertinent physical findings are summarised in the table. The older child (fig 3) was born after 38 weeks' gestation with a birth weight of $3250 \mathrm{~g}$. Head circumference was $36 \mathrm{~cm}$. At birth, an unusual phenotype was noted. The face was elongated, with antimongoloid slanting eyes and corneal opacities. The palate was high and narrow. The forehead was narrow and hairy. The anterior fontanelle was very large and there was a wide metopic suture. The skin and joints were loose and arachnodactyly of the hands and feet was evident. 
A right inguinal hernia was found. With age, joint laxity, arachnodactyly, dorsal kyphosis, and pectus excavatum became prominent. There was a tendency to dislocation of the small joints of the hands and feet. Ocular findings consisted of subluxation of the lens, megalocornea, and blue sclerae. At the age of 3 months, a holosystolic murmur grade $3 / 6$ was first heard at the cardiac apex and radiated to the

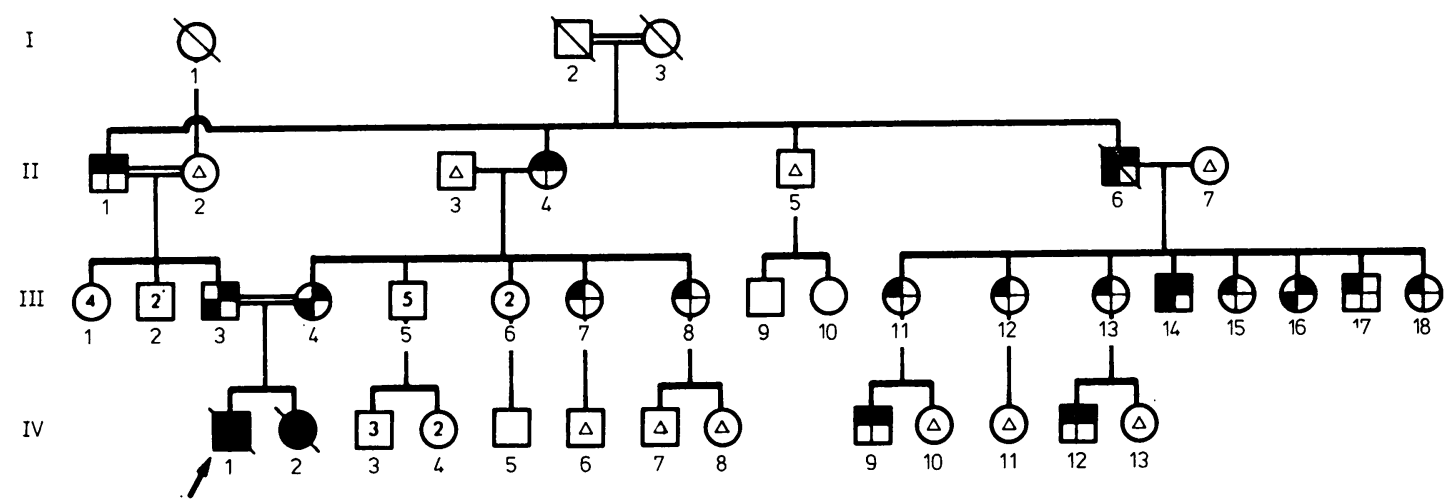

$\boxplus \oplus$ Arachnodactyly
$\boxplus \oplus$ Thoracic deformity
$\boxplus \oplus$ Cardiovascular disease

$\boxplus \oplus$ Ectopia lentis

$\Delta$ (@) Examined, normal

FIG 1 Family pedigree.
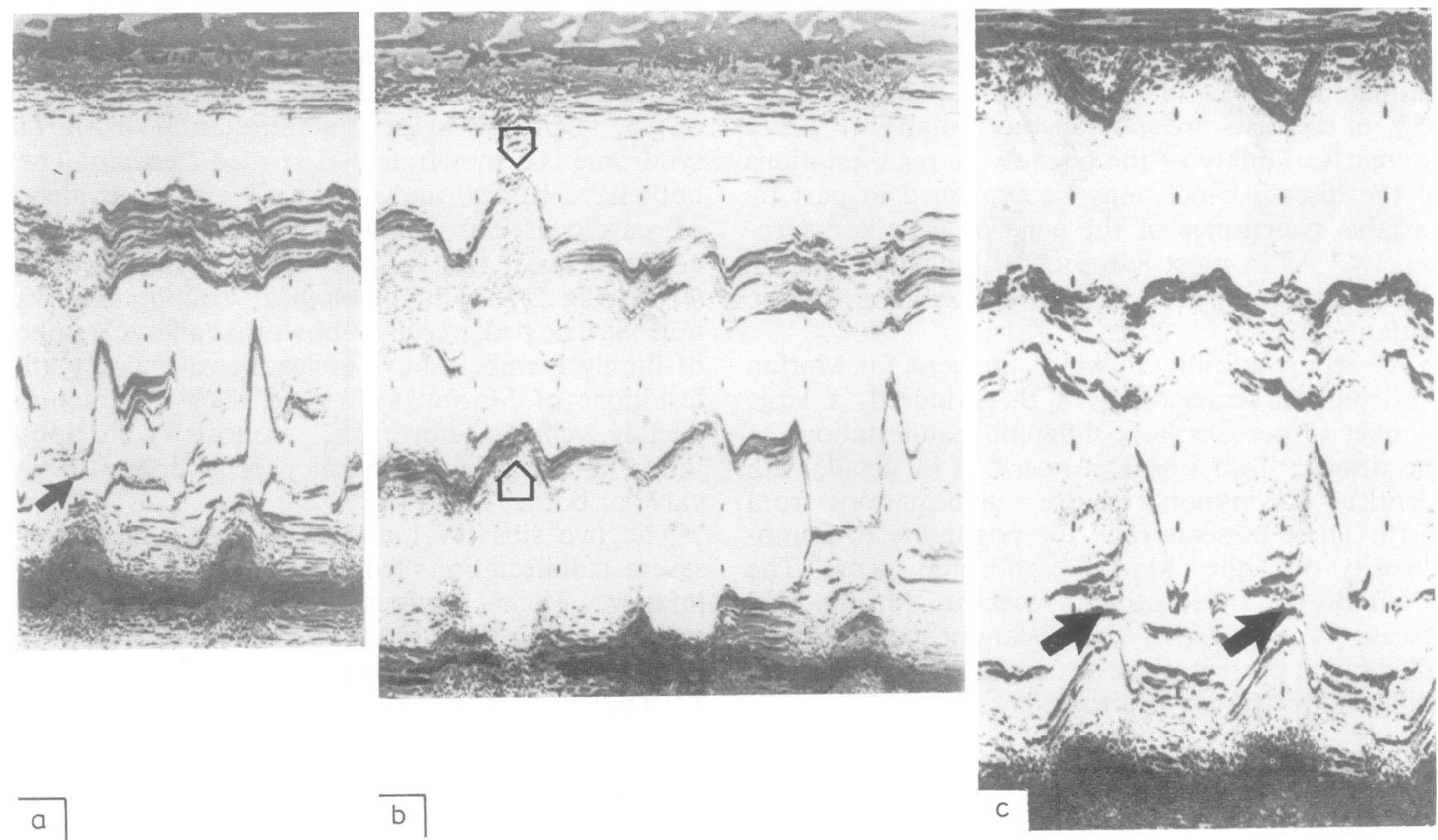

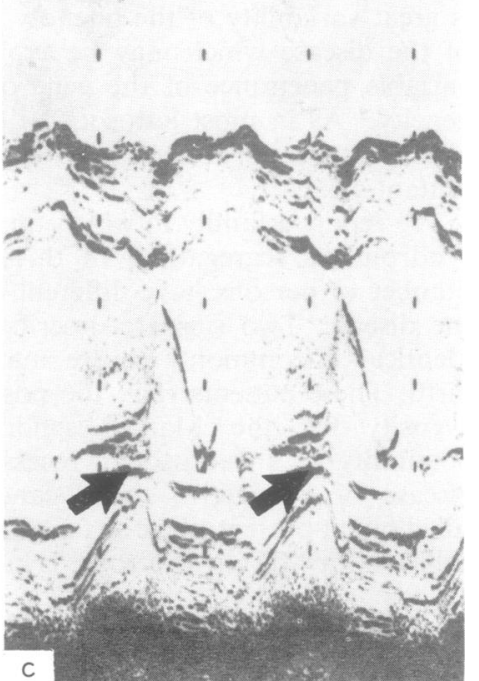

C

FIG 2 M-mode echocardiography of the patients' parents. (a) III.3, left ventricle and mitral valve. Arrow: prolapse of posterior mitral leaflet. (b) III.3, left ventricular outflow tract and aortic valve region. Arrows: enlarged aortic root. (c) III.4, left ventricle and mitral valve region. Arrows: slight prolapse of the posterior valve region. 
TABLE Pertinent physical findings in patients IV.I and IV.2.

\begin{tabular}{ll}
\hline $\begin{array}{l}\text { At birth } \\
\text { Skin } \\
\text { Joints }\end{array}$ & $\begin{array}{c}\text { Loose, not elastic } \\
\text { Dislocations, hypermobility of fingers, } \\
\text { contracture of hips and elbows }\end{array}$ \\
$\begin{array}{l}\text { Skeletal } \\
\text { Hernias }\end{array}$ & $\begin{array}{c}\text { Arachnodactyly, camptodactyly, chest deformity } \\
\text { Inguinal, umbilical }\end{array}$ \\
$\begin{array}{l}\text { Early infancy } \\
\text { Cardiac }\end{array}$ & $\begin{array}{c}\text { Severe and progressive mitral regurgitation, } \\
\text { progressive cardiac and coronary insufficiency }\end{array}$ \\
$\begin{array}{c}\text { Pulmonary } \\
\text { Emphysema, spastic bronchitis, recurrent } \\
\text { pulmonary infections }\end{array}$ \\
Ocular & \begin{tabular}{c} 
Ectopia lentis, megalocornea \\
\hline
\end{tabular}
\end{tabular}

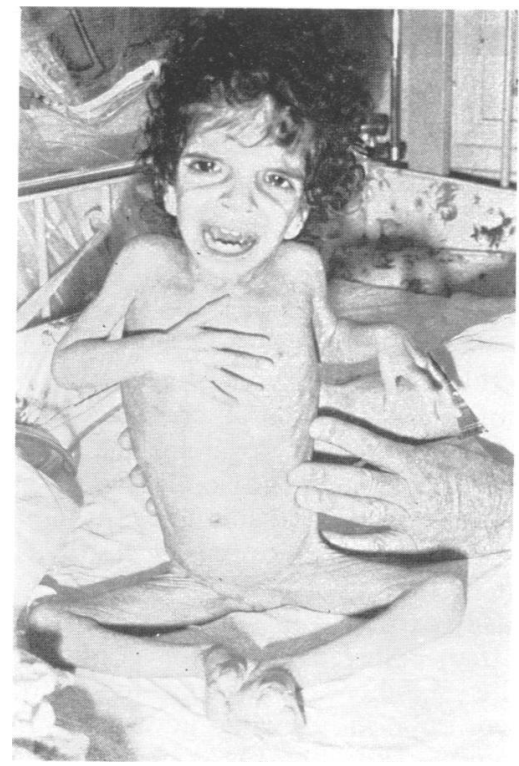

FIG 3 General appearance of the proband. Note facial features, loose skin, arachnodactyly, joint contractures, and chest deformity.

axilla. Cardiological evaluations revealed progressive mitral insufficiency with prolapse of the mitral leaflets. Chest radiographs showed cardiomegaly (fig 4). Repeated echocardiography showed progressive dilation of the aortic root, and systolic flutter and pansystolic prolapse of both mitral leaflets (fig 5). He suffered from recurrent respiratory infections and progressive cardiac insufficiency. At 15 months of age cardiac catheterisation confirmed the presence of severe antral dilation and severe dilation of the aortic sinuses. He developed chest pains and electrocardiographic evidence of anterolateral myocardial infarction. He died at $2 \frac{1}{2}$ years of age at the time of open heart surgery for mitral valve replacement. Mental development was normal.

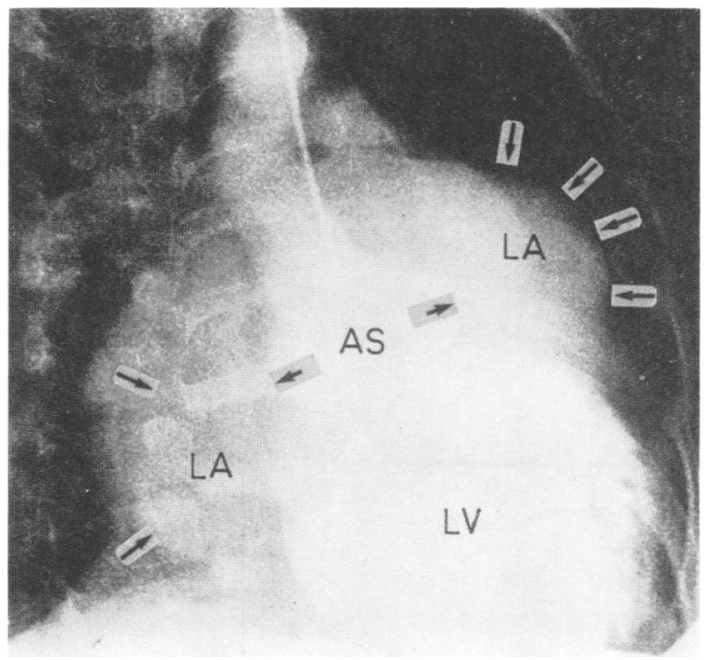

FIG 4 Selective left ventriculography of proband.

Small arrows: enlarged left ventricle $(L V)$ and ectasia of aortic sinuses $(A S)$. Large arrows: huge left atrium $(L A)$ owing to mitral regurgitation.

Pathological examination showed dissection of the pulmonary artery above the pulmonary sinuses. There was severe mucoid degeneration of the mitral and tricuspid valves and a thin and partially disorganised elastic network.

The clinical course and findings in the younger sib were practically identical. She was born after an uneventful term pregnancy weighing $3080 \mathrm{~g}$. Birth length was $51 \cdot 5 \mathrm{~cm}$ and head circumference $36.5 \mathrm{~cm}$. She had very long limbs, a high palate, arachnodactyly, marked pectus excavatum, and joint and skin laxity. A holosystolic murmur was noted from birth. Echocardiography showed prolapsed mitral leaflets. Radiographs showed a markedly enlarged left ventricle. The lenses were dislocated. She developed progressive cardiac failure from 6 months of age and recurrent respiratory tract infections with spastic components. Cardiac catheterisation at the age of 2 years revealed severe mitral insufficiency with prolapse of the mitral valve and aortic ectasia with dilation of the aortic root. She too died at $2 \frac{1}{2}$ years of age of cardiac failure. No necropsy could be performed.

Numerous laboratory tests, including amino-acid chromatography and cytogenetic studies, revealed no abnormalities, excluding those related to the acute phases of the disease at its different stages. Collagen biosynthesis was studied on fibroblasts grown in tissue culture from a skin biopsy obtained from the proband, with the use of radioactive 


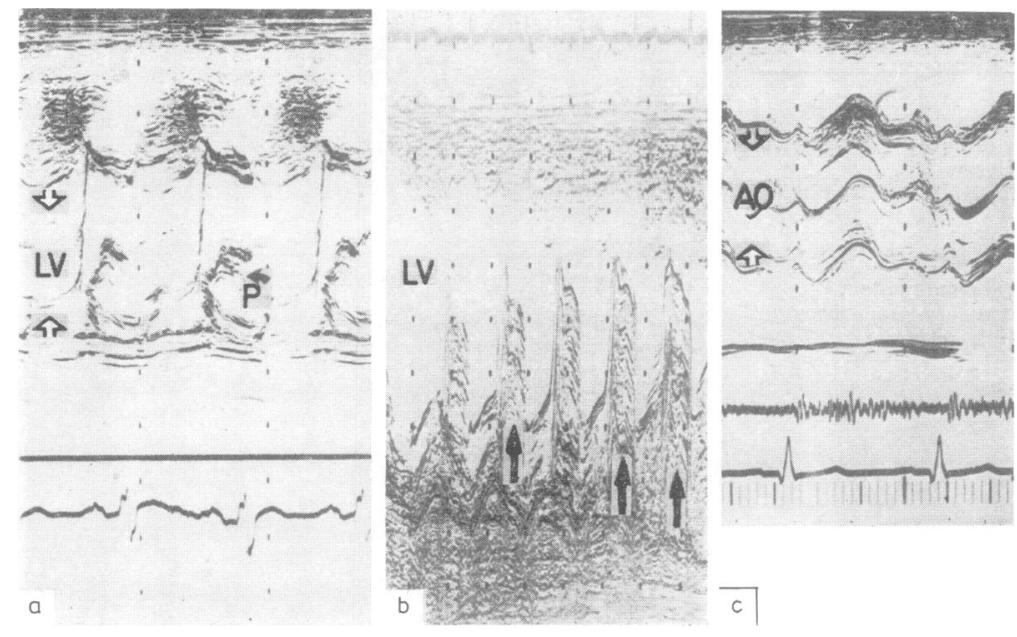

FIG 5 M-mode echocardiography of proband. (a) Enlarged left ventricle (open arrows, $L V$ ), fluttering of posterior $(P)$ mitral leaflet owing to ruptured chordae tendineae, and mitral valve prolapse (arrow). (b) Left ventricle $(L V)$ with multiple echoes (arrows) within mitral valve producing a 'tumour-like' appearance owing to redundant mitral valve. (c) Enlarged aortic root (open arrows, $A O$ ). proline and mannose, protein precipitation by trichloroacetic acid, ${ }^{3}$ slab gel electrophoresis, and fluorescent autoradiography. ${ }^{4}$ The total quantity of collagenous proteins was determined in the medium fraction after dialysis against distilled water and lyophilisation. ${ }^{3}$ The collagen content of the growth medium from the proband's fibroblasts was $3 \cdot 2 \%$ compared to an average of $3.0 \%$ in medium from two age and sex matched control fibroblast cultures. The patterns of fibronectin and procollagens types I and III from the fibroblast culture medium of the proband were very similar to those of control cultures, as well as the extracellular enzymatic conversion of procollagen type I. No further investigations were possible for technical reasons.

\section{Discussion}

Marfan syndrome is an autosomal dominant disease in which a defect in the biosynthesis of collagen is probably involved, or a structural alteration in some other category of connective tissue. ${ }^{15}$ As in other autosomal dominant traits, variable expressivity of the gene results in a wide range of clinical manifestations, possibly as a consequence of variable gene penetrance and genetic heterogeneity. Therefore, the actual prevalence of Marfan syndrome is unknown and may be much greater than the estimated 4 to 6 in 100000 . Affected subjects are usually heterozygotes for the mutant gene. ${ }^{2}$

Homozygosity for autosomal dominant traits is relatively rare in human genetics, and its phenotypic expression is frequently much more severe than that of the heterozygous state. Examples of such cases include achondroplasia, the moderate type of autosomal dominant brachydactyly, the $\vec{\ominus}$ Pelger-Huet anomaly of leucocytes, hereditary $\stackrel{\infty}{\oplus}$ haemorrhagic telangiectasia, and familial hypercholesterolinaemia. ${ }^{6}$

One of the difficulties in characterising Marfan syndrome is the absence of a specific demonstrable $\overline{0}$ defect in the biosynthesis of collagen. Lysyl oxidase $\frac{\circ}{D}$ activity, the enzyme that initiates cross linking, has $\triangle$ been reported to be normal in fibroblasts. ${ }^{7}$ The $\overrightarrow{\overrightarrow{0}}$ collagen in skin fibroblasts of affected subjects is $\overline{3}$ more easily extracted in aqueous and denaturating buffers than collagen in normal controls. ${ }^{89}$ However, the stability of the insoluble fraction is decreased only in growing adolescent patients. ${ }^{10}$ Furthermore, skin fibroblasts from patients with $\frac{0}{3}$ Marfan syndrome synthesise approximately five times more hyaluronic acid than normal human skin $ᄋ$ fibroblasts. ${ }^{11}$ It has been suggested that this is because of a defect in the control of the synthesis of 음 hyaluronic acid ${ }^{12}$ owing to increased activity of the $D$ hyaluronic acid synthetase. ${ }^{13}$ Several alterations in the biosynthesis of collagenous proteins in patients $\bar{N}$ with Marfan syndrome have been described. These $\Omega$ include differences in the proportions of collagen $N$ types $\mathrm{I}$ and $\mathrm{III}^{\mathbf{1 4}}$ and changes in the ultrastructure $\underset{\mathrm{N}}{\mathrm{N}}$ and composition of collagen in aortic tissue. ${ }^{15}$ In the latter case, the aortic tissue contained two distinct 0 $\alpha 2$ chains of collagen type I. Reduced amounts of chemically stable forms of intermolecular cross links $\stackrel{\oplus}{?}$ in skin fibroblasts were recently observed, ${ }^{16}$ and it $\square$ was suggested that the cause of this defective collagen could be in altered maturation. It has been $\stackrel{\mathbb{D}}{\Omega}$ proposed that these cross link abnormalities are $\stackrel{\mathbb{Q}}{\complement}$ secondary to a primary gene defect in the $\alpha 2$ (I) peptide. ${ }^{17}$ Dermal fibroblasts from a patient with 
Marfan syndrome were shown to synthesise two $\alpha 2$ collagen chains of which one contained an insertion of approximately 20 amino-acids. ${ }^{17}$ The $\alpha 2$ (I) genes have been assigned to chromosome 7 by molecular hybridisation. ${ }^{5}$ Biochemical analyses of a large number of Marfan syndrome patients are necessary in order to determine whether the appearance of an abnormal $\alpha 2$ collagen chain is a general and specific defect in this condition.

We propose that the two severely affected sibs represent examples of homozygosity for the Marfan gene. This assumption is based on the severe and similar course of the Marfan-like disease and the presence of Marfan syndrome in both parents in a family in which a large number of persons have this syndrome. Homozygosity for Marfan syndrome may have occurred in a large family described in 1959 and mentioned by McKusick, in which there were some subjects much more severely affected born to first cousin parents. ${ }^{18}$

However, other possible genetic mechanisms have to be considered, even though none can be proven owing to the lack of a specific detectable abnormality of collagen. Fried and Krakowski ${ }^{19}$ postulated autosomal recessive inheritance for Marfan syndrome in a family in which there were two affected sisters in a sibship with completely normal and non-consanguineous parents. In the family presented here, the parents are first cousins and both patients had a similar disease. Therefore, it could be assumed that the patients are homozygous for an autosomal recessive gene. The parents would thus be heterozygous and mildly affected.

Another genetic model could be the presence of double dominant heterozygosity in both patients. Each parent would be heterozygous for an autosomal dominant gene for a different type of abnormal collagen at different loci, and their offspring would thus be heterozygotes for each one of these genes. Mutations at different loci, with alterations of different subunits of collagen, are potentially possible, even though they have not been demonstrated so far.

Until further knowledge is obtained on a specific molecular defect in Marfan syndrome, genetic counselling will be based on theoretical recurrence risk figures. Assuming that the two patients represent homozygosity for the autosomal dominant Marfan syndrome gene, the recurrence risks in each pregnancy would be $25 \%$ and $50 \%$ for homozygous and heterozygous Marfan syndrome, respectively.
This work was supported in part by a grant from the Advancement of Mankind foundation (to DD).

\section{References}

1 Pyeritz RE, McKusick VA. The Marfan syndrome: diagnosis and management. $N$ Engl J Med 1979;300: $772-7$.

2 Pyeritz RE, Murphy EA, McKusick VA. Clinical variability in the Marfan syndrome(s). Birth Defects $1979 ; X V(5 B): 155-78$.

3 Duksin D, Bornstein P. Impaired conversion of procollagen to collagen by fibroblasts and bone treated with tunicamycin, an inhibitor of protein glycosylation. J Biol Chem 1977;252:955-62.

4 Duksin D, Mahoney WC. Relationship of the structure and biological activity of the natural homologues of tunicamycin. J Biol Chem 1982;257:3105-9.

5 Pyeritz RE, McKusick VA. Basic defects in the Marfan syndrome. $N$ Engl J Med 1981 ;305:1011-2.

6 Vogel F, Motulsky AG. Human genetics. Problems and approaches. New York: Springer-Verlag, 1979.

7 Layman DL, Narayanan AS, Martin GR. The production of lysyl oxidase by human fibroblasts in culture. Arch Biochem Biophys 1972;149:97-101.

8 Laitinen $O$, Uitto J, Livanainen $M$, Hannuksela $M$, Kivirikko KI. Collagen metabolism in Marfan's syndrome. Clin Chim Acta 1968 ;21:321-6.

- Priest RE, Moinuddin JF, Priest JH. Collagen in Marfan syndrome is abnormally soluble. Nature 1973;245:264-6.

10 Francis MJO, Sanderson MC, Smith R. Skin collagen in idiopathic adolescent scoliosis and Marfan's syndrome. Clin Sci Mol Med 1976;51:467-74.

11 Matalon R, Dorfman A. The accumulation of hyaluronic acid in cultured fibroblasts of the Marfan syndrome. Biochem Biophys Res Commun 1968;32:150-4.

12 Lamberg SI, Dorfman A. Synthesis and degradation of hyaluronic acid in the cultured fibroblasts of Marfan's syndrome. J Clin Invest 1973;52:2428-33.

13 Appel A, Horwitz AL, Dorfman A. Cell-free synthesis of hyaluronic acid in Marfan syndrome. J Biol Chem 1979; 254:12199-203.

14 Halbritter R, Aumailley M, Rackwitz R, Kreig T, Muller PK. Case report and study of collagen metabolism in Marfan's syndrome. Klin Wochenschr 1981 ;59:83-90.

15 Scheck M, Siegal RC, Parker J, Chang YH, Fu JCC. Aortic aneurism in Marfan's syndrome: changes in the ultrastructure and composition of collagen. J Anat 1979; 129:645-57.

16 Boucek RJ, Noble NL, Gunja-Smith Z, Butler WT. The Marfan syndrome: a deficiency in chemically stable collagen cross-links. $N$ Engl J Med $1981 ; 305: 988-91$.

17 Byers PH, Siegal RC, Peterson KE, et al. Marfan syndrome: abnormal $\alpha 2$ chain in type I collagen. Proc Natl Acad Sci USA 1981 ;78:7745-9.

18 McKusick VA. Heritable disorders of connective tissue. St Louis: Mosby, 1972.

19 Fried K, Krakowsky D. Probable autosomal recessive Marfan syndrome. J Med Genet 1977;14:359-61.

Correspondence and requests for reprints to Dr Juan Chemke, Clinical Genetics Unit, Kaplan Hospital, 76100 Rehovot, Israel. 